\title{
PRS-on-Spark (PRSOS): a novel, efficient and flexible approach for generating polygenic risk scores
}

Lawrence M. Chen ${ }^{1,2}$, Nelson Yao ${ }^{1,2}$, Elika Garg ${ }^{1,2}$, Yuecai Zhu ${ }^{1,2}$, Thao T. T. Nguyen 1,2, Irina Pokhvisneva ${ }^{1,2}$, Shantala A. Hari Dass ${ }^{1,2}$, Eva Unternaehrer ${ }^{1,2}$, Hélène Gaudreau ${ }^{1,2}$, Marie Forest ${ }^{2,3}$, Lisa M. McEwen ${ }^{4}$, Julia L. Maclsaac ${ }^{4}$, Michael S. Kobor ${ }^{4}$, Celia M. T. Greenwood ${ }^{2,3,5,6,7}$, Patricia P. Silveira ${ }^{1,2,8,9}$, Michael J. Meaney ${ }^{1,2,8,9,10,11}$ and Kieran J. O'Donnell ${ }^{1,2,8,9,10^{*}}$

\begin{abstract}
Background: Polygenic risk scores (PRS) describe the genomic contribution to complex phenotypes and consistently account for a larger proportion of variance in outcome than single nucleotide polymorphisms (SNPs) alone. However, there is little consensus on the optimal data input for generating PRS, and existing approaches largely preclude the use of imputed posterior probabilities and strand-ambiguous SNPs i.e., A/T or C/G polymorphisms. Our ability to predict complex traits that arise from the additive effects of a large number of SNPs would likely benefit from a more inclusive approach.

Results: We developed PRS-on-Spark (PRSOS), a software implemented in Apache Spark and Python that accommodates different data inputs and strand-ambiguous SNPs to calculate PRS. We compared performance between PRSOS and an existing software (PRSice v1.25) for generating PRS for major depressive disorder using a community cohort ( $N=264$ ). We found PRSoS to perform faster than PRSice v1.25 when PRS were generated for a large number of SNPs ( $\sim 17$ million SNPs; $t=42.865, p=5.43 \mathrm{E}-04)$. We also show that the use of imputed posterior probabilities and the inclusion of strand-ambiguous SNPs increase the proportion of variance explained by a PRS for major depressive disorder (from $4.3 \%$ to $4.8 \%$ ).

Conclusions: PRSOS provides the user with the ability to generate PRS using an inclusive and efficient approach that considers a larger number of SNPs than conventional approaches. We show that a PRS for major depressive disorder that includes strand-ambiguous SNPs, calculated using PRSOS, accounts for the largest proportion of variance in symptoms of depression in a community cohort, demonstrating the utility of this approach. The availability of this software will help users develop more informative PRS for a variety of complex phenotypes.
\end{abstract}

Keywords: PRS-on-spark, PRSoS, Polygenic risk score, Genetic profile score, Multi-core processing, Bioinformatics, Major depressive disorder

\footnotetext{
* Correspondence: kieran.odonnell@mcgill.ca

'Douglas Hospital Research Centre, McGill University, H4H1R3, Montreal,

Quebec, Canada

${ }^{2}$ Ludmer Centre for Neuroinformatics and Mental Health, McGill University,

Montreal, QC, Canada

Full list of author information is available at the end of the article
}

(c) The Author(s). 2018 Open Access This article is distributed under the terms of the Creative Commons Attribution 4.0 International License (http://creativecommons.org/licenses/by/4.0/), which permits unrestricted use, distribution, and reproduction in any medium, provided you give appropriate credit to the original author(s) and the source, provide a link to the Creative Commons license, and indicate if changes were made. The Creative Commons Public Domain Dedication waiver (http://creativecommons.org/publicdomain/zero/1.0/) applies to the data made available in this article, unless otherwise stated. 


\section{Background}

Polygenic risk scores (PRS) provide an index of the cumulative contribution of common variants to complex traits [1]. The approach has been applied to a large number of phenotypes, including height [2], body mass index [3], and disease risk, most notably in the prediction of psychiatric disorders [4-6]. PRS build on large existing discovery genome-wide association studies (GWAS), such as those provided by the Psychiatric Genomics Consortium (PGC) [7], which provide weights (odds ratios for binary outcomes and beta coefficients for continuous traits) that index the association between a single nucleotide polymorphism (SNP) and a phenotype of interest. Thus PRS are given by:

$$
P R S=\sum_{i=1 \ldots x}^{n} \beta_{i} \cdot G_{i}
$$

Where $\beta_{\mathrm{i}}=$ the natural logarithm of the odds ratio (or beta coefficient) between the "ith" SNP and phenotype of interest and $G_{i}=$ allele count (e.g., 0,1,2) at the "ith" SNP.

PRS calculations are memory intensive, due to the large number of SNPs considered in each PRS. PLINK $[8,9]$ can be used to calculate PRS quickly. However, datasets must first undergo a series of pre-processing steps. PRSice v1.25 [10] is a software that simplifies this process through semi-automation. It is written in $\mathrm{R}$ and uses PLINK $[8,9]$ to calculate PRS. PRSice v1.25 uses observed genotypes or imputed posterior probabilities that have been converted to best guess genotypes ("hard calls") to calculate PRS. It can also accommodate imputed posterior probabilities but relies on a dated and slower version of PLINK (version 1.07) [9]. Likewise, PRSice v1.25 discards strand-ambiguous SNPs, which are SNPs that have A/T or C/G alleles. If the strand assignment of the strand-ambiguous SNP is unknown, misassignment can occur (see Fig. 1). Many GWAS do not report the reference strand, which can lead to ambiguity on the identification of the "risk/effect allele" and the corresponding weight that should be applied. Consequently, strand-ambiguous SNPs are typically removed prior to PRS calculations (e.g., [11-15]).

As a solution we propose to use allele frequency information that many GWAS report (e.g., PGC [16, 17], GIANT [18], STARRS [19]) to identify the "effect" allele across datasets. The inclusion of the strand-ambiguous SNPs would allow researchers to retain as much information as possible from the discovery data, and likely give rise to a better understanding of the complex phenotypes.

We have developed a new software package, PRS-onSpark (PRSoS), which accommodates observed genotypes or imputed posterior probabilities. Further, it includes a novel function that retains strand-ambiguous SNPs by using allele frequency data to identify the effect allele between discovery and target datasets. Here we test the performance of PRSoS against PRSice v1.25 using genetic data derived from a Canadian cohort and demonstrate the enhanced predictive power of PRS generated from PRSoS in the prediction of symptoms of depression.

\section{Implementation}

PRS-on-Spark (PRSoS: https:/github.com/MeaneyLab/ PRSoS) is implemented in Apache Spark 2.0.0+ (Spark) and Python 2.7. Spark is an open source cluster-computing framework for big data processing that can be integrated into Python programming. As such, Spark facilitates data partitioning and parallel processing across multiple nodes and cores. For the current analyses we ran PRSoS on Linux CentOS 7, 24-core Intel Xeon server with 256GB RAM, using Spark standalone mode and a distributed file system (Apache Hadoop) with 12 cores across one worker (maximum available $\mathrm{RAM}=48 \mathrm{~GB}$ ). PRSoS can also be implemented as a standalone version on a single cluster. PRSoS runs on the command line in Terminal on Linux or Mac, or Command Prompt in Windows. PRSoS is currently compatible with both Oxford genotype files (.gen/ .sample) and Variant Call Format (VCF) files.

Equation 2 describes our approach to PRS calculation that accommodates imputed posterior probabilities typical of imputed genetic data:

$$
P R S=\sum_{i=1 \ldots x}^{n}\left(2 \beta_{i} \cdot p\left(A A_{i}\right)+1 \beta_{i} \cdot p\left(A B_{i}\right)+0 \beta_{i} \cdot p\left(A A_{i}\right)\right.
$$

Which gives:

$$
P R S=\sum_{i=1 \ldots x}^{n}\left(2 \beta_{i} \cdot p\left(A A_{i}\right)+\beta_{i} \cdot p\left(A B_{i}\right)\right)
$$

Which gives:

$$
P R S=\sum_{i=1 \ldots x}^{n} \beta_{i} \cdot\left(2 p\left(A A_{i}\right)+p\left(A B_{i}\right)\right)
$$

where $\mathrm{p}\left(\mathrm{AA}_{\mathrm{i}}\right)$ is the probability of a homozygous genotype for the effect allele (AA) at the $\mathrm{i}^{\text {th }} \mathrm{SNP}, \mathrm{p}\left(\mathrm{AB}_{\mathrm{i}}\right)$ is the probability of a heterozygous genotype with one copy of the effect allele at the $\mathrm{i}^{\text {th }}$ SNP and $\mathrm{p}\left(\mathrm{BB}_{\mathrm{i}}\right)$ is the probability of a homozygous genotype with zero copies of the effect allele i.e., BB genotype. This approach (Eq. 2) can also be used with observed genotypes and hard calls to calculate PRS. In the final step PRS are divided by the SNP count to obtain the weighted average across the number of SNPs called for each sample, which can vary across participants. These SNP counts for each sample are also provided in the PRSoS output.

PRSoS implements an allele frequency function to match the target dataset's allele to the effect allele 


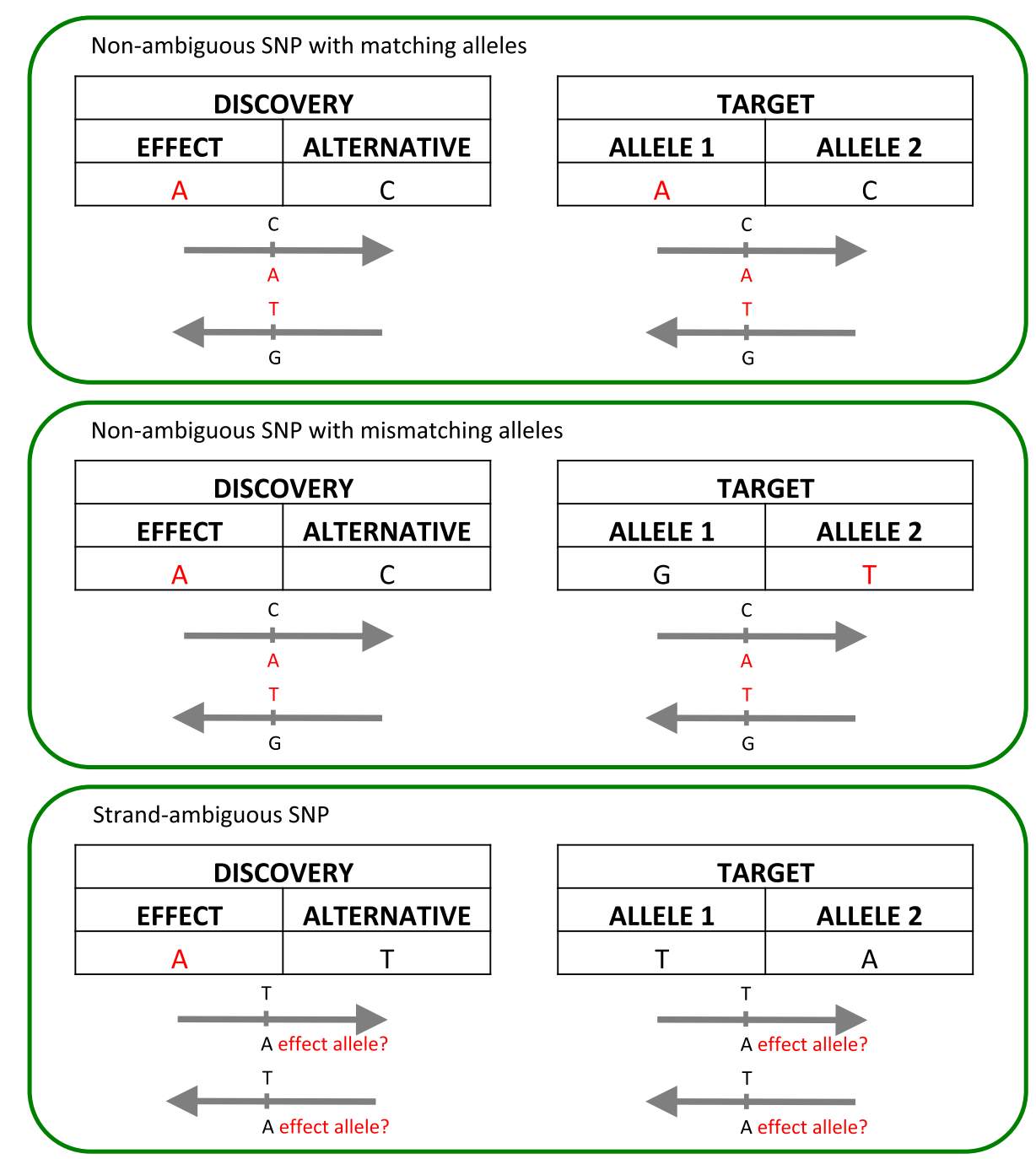

Fig. 1 Allele matching for polygenic risk scores (PRS) between discovery and target data. The effect alleles and their reverse complements are indicated in red. Matching the effect alleles from the discovery data with the reported alleles in the target data is straightforward when SNPs are not strand-ambiguous (top and middle panel). The allele in the target data can be misassigned for strand-ambiguous SNPs (bottom)

reported in the discovery GWAS for ambiguous SNPs i.e., A/T or $\mathrm{C} / \mathrm{G}$ allelic pairs. Note, this function does not explicitly identify strandedness (e.g., forward nor reverse strand) or perform strand alignment, rather it tests if the allele frequency of the effect allele (in the discovery dataset) matches the allele frequency for a given allele in the target dataset. The function will discard strand-ambiguous SNPs with an allele frequency between 0.4 and 0.6 , to ensure alleles can be matched with a high degree of certainty. If both the allele frequencies are less than 0.4, then the first allele in the target data is scored. Likewise, if both the allele frequencies are greater than 0.6, then the first allele in the target data is scored. In contrast, if only one of the allele frequencies is less than 0.4 (while the other is greater than 0.6) then the second allele in the target data is scored (Fig. 2). This setting can be disabled (e.g., when the allele frequency in the discovery data is not provided). We note that this approach is best suited to discovery/target datasets that have a similar population structure and should not be applied to datasets with marked differences in ethnicity across cohorts.

PRSoS can also provide a SNP log documenting the SNPs included in a PRS at any given $p$-value threshold (Table 1). This SNP list can be used for subsequent gene ontology, pathway, or network analysis. The SNP log also identifies SNPs that have been excluded from the PRS, for example, when alleles in the discovery data and the target data are not identical, such as for multi-allelic SNPs.

\section{Sample data and polygenic risk scoring}

We used genotype and phenotype data from the Maternal Adversity, Vulnerability and Neurodevelopment (MAVAN) 


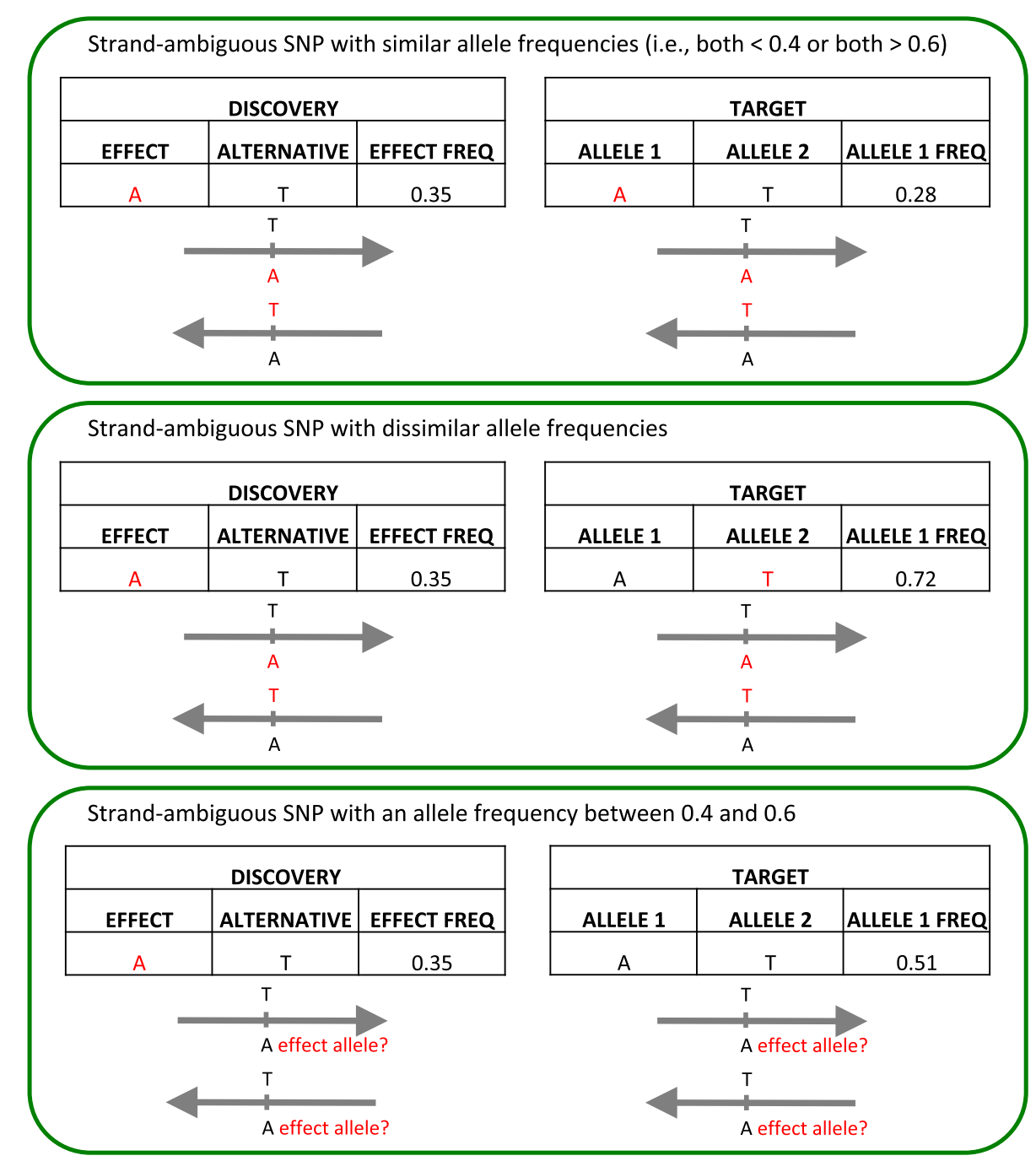

Fig. 2 PRSOS allele matching solution for strand-ambiguous SNPS. The effect alleles and their reverse complements are indicated in red. The discovery effect allele and the target allele 1 are the same if their allele frequencies are both less than 0.4 or both more than 0.6 (top). The target allele 1 is not the effect allele if one has low allele frequency and the other has high allele frequency (middle). Strand-ambiguous SNPs with an allele frequency between 0.4 and 0.6 are excluded to increase the certainty of matching alleles

study [20] as our target dataset. Details about the inclusion, selected measures, genotyping, quality control, and imputation are described in Additional file 1. Depressive symptoms were assessed using a well-validated, standardized questionnaire [21]. Table 2 provides a summary of the subsample used. We used the PGC major depressive disorder (MDD) GWAS as our discovery data [16]. We used the PGC MDD clumped file (pgc.mdd.clump.2012-04.txt) for all analyses. Clumping uses a greedy algorithm to selectively prune SNPs within regions of linkage disequilibrium based on the association $p$-value between each SNP and the phenotype e.g., MDD of interest [22].

\section{Performance analysis}

We compared the performance (processing times in seconds) of PRSice v1.25 and PRSoS across three types of data input: 1) imputed posterior probabilities (Imputed PP); 2) imputed genotypes converted to hard calls (Imputed $\mathrm{HC}$ ), and 3) observed genotypes (Array Data). However, PRSice v1.25 and PRSoS are best-suited for different file formats: PLINK (.bed/.bim/.fam) format and Oxford (.gen/.sample) format, respectively. Further, .bed/.bim/.fam files are not compatible with imputed posterior probabilities. Therefore, we first compared PRSice v1.25 and PRSoS using the same format (Oxford files) for the Imputed PP. Thereafter, we compared PRSice v1.25 and PRSoS using their optimal formats for the other two data inputs (PRSice $=$.bed/.bim/.fam and $\mathrm{PRSoS}=$.gen/.sample). We used PRSoS and PRSice v1.25 to calculate PRS at five $p$-value thresholds $\left(\mathrm{P}_{\mathrm{T}}=0.1\right.$, $0.2,0.3,0.4,0.5)$ in a single run for each data input. Strand-ambiguous SNPs were not considered in this test. 
Table 1 PRSOS optional data output

\begin{tabular}{lllll}
\hline PRS_0.001 & PRS_0.001_flag & PRS_0.5 & PRS_0.5_flag & Discard \\
\hline rs1115507 & A1 & rs1115507 & A1 & rs2503243 \\
rs17692694 & A2 & rs11661323 & A2 & rs519113 \\
rs4544201 & A2 & rs12296077 & A1 & \\
rs6683133 & A1 & rs12611811 & A1 & \\
rs7609940 & A2 & rs17024456 & A1 & \\
rs7620685 & A1 & rs17692694 & A2 & \\
& & rs4544201 & A2 & \\
& & rs6683133 & A1 & \\
& & rs7609940 & A2 & \\
& & rs7620685 & A1 &
\end{tabular}

Example of the SNP log included in the PRSoS output. The SNP log records the SNPs that are used in the PRS at each p-value threshold and whether the first allele column ("A1") or the second allele column ("A2") in the target data was scored. SNPs are recorded in the Discard column if the SNPs are discarded due to non-matching alleles between the discovery and the target data

We performed this calculation three times for each software. We used a paired $\mathrm{t}$-test to describe differences in total processing time. In addition, we tested if the optional SNP $\log$ available in PRSoS (see Table 1) significantly increases PRS computation time. Table 3 provides a summary of the genotype data input. All PRSoS calculations were performed using 12 physical cores in our server, with one thread of execution per core. PRSice v1.25 does not have a multi-thread option; thus it used one thread on one core. In a supplementary analysis, we illustrate the enhanced performance of PRSoS across an increasing number of cores (see Additional file 2: Fig. S1).

We also tested the performance of PRSice v1.25 and PRSoS at an increasing number of $p$-value thresholds. Specifically, we used the Imputed HC to generate PRS at $5,10,25,50,100,125$, or $200 p$-value thresholds $\left(\mathrm{P}_{\mathrm{T}}\right.$ range: $\left.0-0.5\right)$. See Additional file 1 for the

Table 2 Maternal Adversity, Vulnerability and Neurodevelopment (MAVAN) cohort demographics. Symptoms of depression were assessed using the Center for Epidemiological Studies - Depression (CES-D) scale

\begin{tabular}{|c|c|}
\hline \multicolumn{2}{|l|}{ Cohort Demographics } \\
\hline \multicolumn{2}{|l|}{ Sample size } \\
\hline Genotyping data only (used in software performance test) & $N=264$ \\
\hline Genotyping data with symptoms of depressive score (CES-D) & $N=236$ \\
\hline Mean age at time of assessment in years (SD) & $34.65(4.89)$ \\
\hline Mean symptoms of depressive score (SD) & $10.07(8.81)$ \\
\hline \multicolumn{2}{|c|}{ Reported ethnicity among sample with genotyping data and CES-D data } \\
\hline Caucasian & $N=201$ \\
\hline Others & $N=34$ \\
\hline Not reported & $N=1$ \\
\hline
\end{tabular}

Table 3 Genotyping file information

\begin{tabular}{lllll}
\hline \multicolumn{1}{c}{} & & $\begin{array}{l}\text { Genotyping file } \\
\text { format }\end{array}$ & $\begin{array}{l}\text { File size } \\
\text { (GB) }\end{array}$ & $\begin{array}{l}\text { SNP } \\
\text { count }\end{array}$ \\
\hline PRSice v1.25 & Array Data & .bim/.bed/fam & 0.03 & 316,480 \\
& $\begin{array}{l}\text { Imputed } \\
\text { HC }\end{array}$ & .bim/.bed/fam & 1.66 & $17,434,284$ \\
& Imputed & .gen/fam & 29.02 & $17,434,284$ \\
& PP & & & \\
PRSoS & Array Data & .gen/.sample & 0.51 & 316,480 \\
& Imputed & .gen/.sample & 28.09 & $17,434,284$ \\
& HC & & & \\
& Imputed & .gen/.sample & 29.02 & $17,434,284$ \\
& PP & & & \\
\hline
\end{tabular}

The file size and SNP count provide an idea of how much data processing needs to be done by each software in our analysis. The file formats that we used in PRSice and PRSoS are different due to differences in file compatibility. All files have the same sample size $(\mathrm{N}=264)$

executable commands used for these comparisons. Additional optional features in PRSice v1.25 (i.e., clumping and regression analysis) and PRSoS (i.e., the SNP $\log$ ) were disabled in the tests to ensure that the comparisons focused solely on PRS computation.

Finally, we used PRSoS and each of the three datasets (i.e., Imputed PP, Imputed HC, Array Data) to test if the inclusion of strand-ambiguous SNPs increased the predictive value of PRS for MDD. We used linear models and compared the proportion of variance explained by PRS with and without strand-ambiguous SNPs.

\section{Results}

PRSoS calculated PRS $\left(\mathrm{P}_{\mathrm{T}}=0.1,0.2,0.3,0.4,0.5\right)$ using the Imputed PP in $169.6 \mathrm{~s}(\mathrm{SD}=0.93 \mathrm{~s})$. The same calculation using PRSice v1.25 took $8461.3 \mathrm{~s}(\mathrm{SD}=334.6 \mathrm{~s})$, which was significantly longer than PRSoS $(t=42.865$, $p=5.43 \mathrm{E}-04$, two-tailed; Fig. 3). Figure 3 also shows the performance of PRSice v1.25 and PRSoS using the Imputed HC and Array Data. PRSoS calculated PRS more quickly than PRSice v1.25 when using the Imputed HC $(t=62.627, p=2.55 \mathrm{E}-04$, two-tailed) but not when using the smaller Array Data $(t=-24.978, p=1.60 \mathrm{E}-03$, two-tailed), where PRSice v1.25 performed best. The addition of the SNP log output did not significantly increase processing times.

\section{The number of $p$-value thresholds affects PRSoS performance}

PRSice v1.25 provides a "high-resolution" option, creating PRS at a large number of $\mathrm{p}$-value thresholds in a single run. We tested the performance of PRSoS against PRSice v1.25 at different resolutions (up to $200 p$-value thresholds) using the Imputed HC. PRSice v1.25 took $0.09 \mathrm{~s}(\mathrm{SD}=0.07 \mathrm{~s})$ to calculate PRS for each threshold in addition to $795.7 \mathrm{~s}(\mathrm{SD}=6.6 \mathrm{~s})$ for other processing 


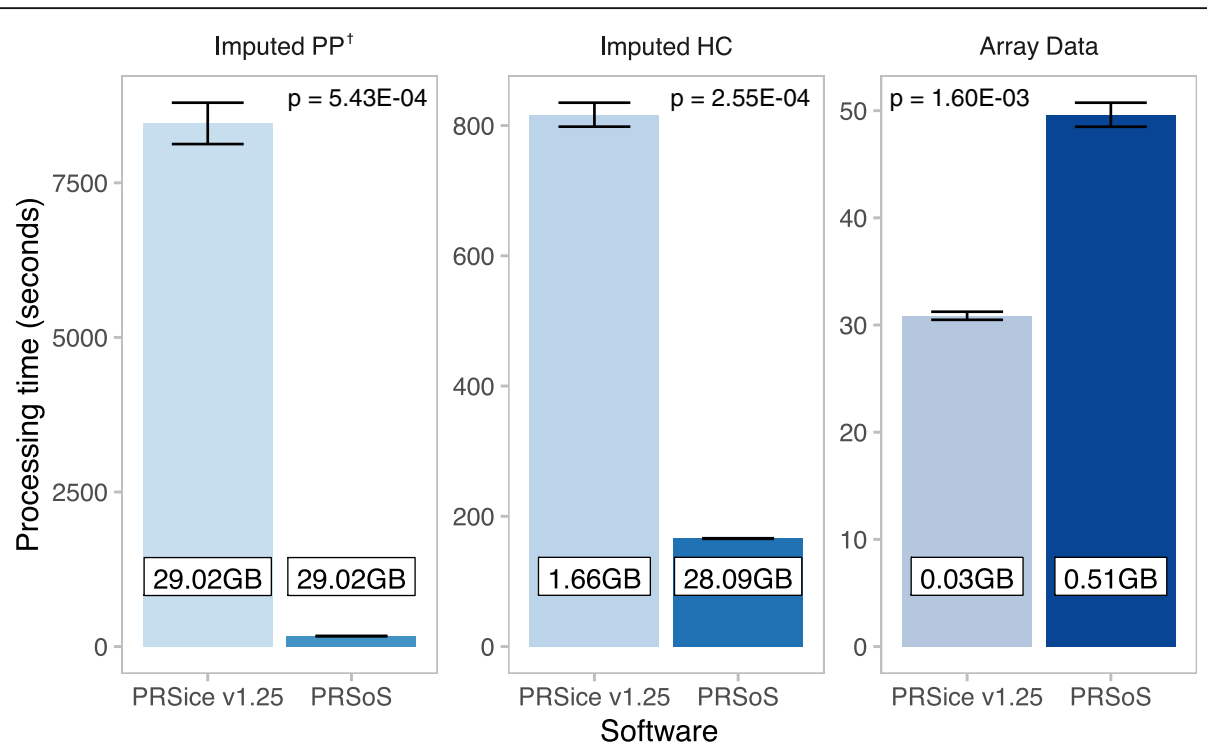

Fig. 3 PRSice v1.25 and PRSOS performance across datasets. Bar plot shows the results of the performance test comparing running PRSice v1.25 and PRSOS across the datasets. Error bars indicate standard deviations. Numbers in boxed inserts indicate the size of the genotype data input. ${ }^{\dagger}$ Note that the file sizes used for the Imputed PP are same for PRSice v1.25 and PRSOS, thus illustrating the processing speed difference with same file size input. Imputed PP = imputed posterior probabilities, Imputed HC = imputed posterior probabilities converted to "hard calls", Array Data = observed genotypes. Significance values derived from paired t-tests

operations (e.g., reading data). PRSoS processing times increased linearly with the number of thresholds (intercept $=156.8 \mathrm{~s}$, slope $=2.14 \mathrm{~s} /$ threshold $)$. PRSoS took $2.14 \mathrm{~s}(\mathrm{SD}=0.04 \mathrm{~s})$ to calculate PRS for each additional threshold in addition to $156.8 \mathrm{~s}(\mathrm{SD}=4.1 \mathrm{~s})$ for other processing operations. Although PRSoS took longer to calculate PRS for a single threshold, PRSoS calculated PRS more quickly than PRSice v1.25 in all other comparisons (Fig. 4).

\section{Strand-ambiguous SNPs explain additional variance in phenotype}

We sought to determine the optimal data input (i.e., Array Data, Imputed HC, and Imputed PP datasets with and without strand-ambiguous SNPs) to derive PRS that accounted for the largest proportion of variance in symptoms of MDD. We observed a positive association between PRS for MDD and depressive symptoms across all datasets (Fig. 5) however the "best-fit" $p$-value threshold varied

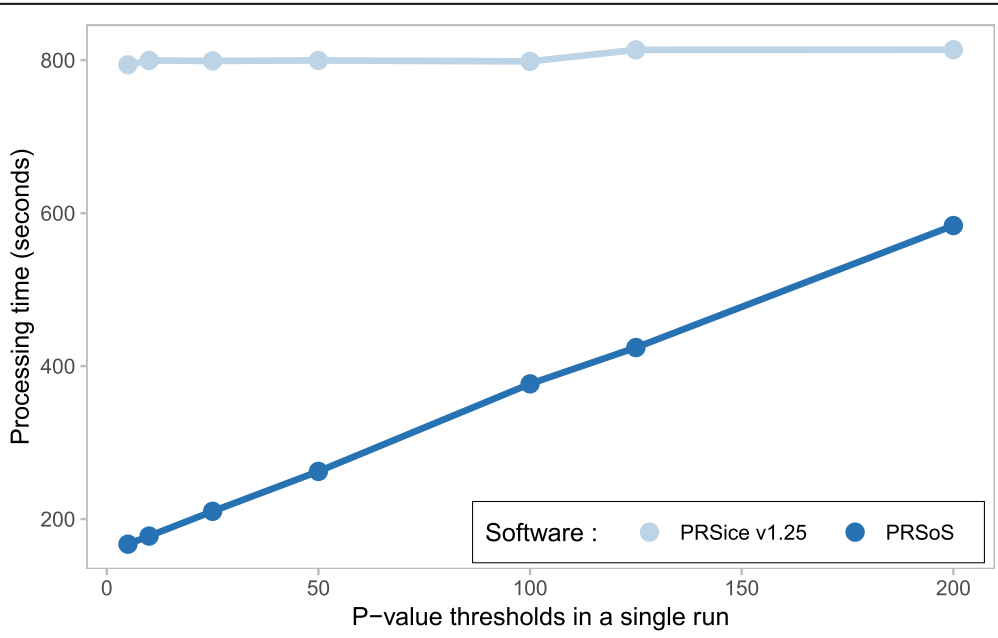

Fig. 4 PRSice v1.25 and PRSoS performance across increasing number of $p$-value thresholds. Line plot shows the results of the performance test comparing PRSice 1.25 and PRSOS across increasing number of p-value thresholds to construct in a single run using a dataset based on imputed posterior probabilities converted to "hard calls" (Imputed HC) 
across different datasets. For example, the PRS at $\mathrm{P}_{\mathrm{T}}=0.2$ accounted for the largest proportion of variance of all PRS generated from the Array Data. In contrast, the PRS at $\mathrm{P}_{\mathrm{T}}=0.1$ performed best for both the Imputed $\mathrm{HC}$ and Imputed PP. PRS generated from the Imputed PP that included strand-ambiguous SNPs accounted for the largest proportion of variance in depressive symptoms $\left(R^{2}=0.048, \quad F \quad(1,234)=11.88\right.$, $p=6.71 \mathrm{E}-04)$. In all models, the inclusion of strand-ambiguous SNPs increased the proportion of variance explained by PRS for MDD (Fig. 6).

\section{Discussion}

PRS-on-Spark (PRSoS) is a flexible and efficient software for generating PRS. We show that PRSoS, which makes use of parallel computing, outperforms PRSice v1.25 when using imputed posterior probabilities (Imputed PP) at a number of $p$-value thresholds. We also show that PRSoS accommodates strand-ambiguous SNPs, which increase the proportion of variance explained by a PRS for MDD.

This is the first report to date comparing different strategies for calculating PRS for MDD. Our analyses demonstrate that a PRS based on imputed posterior probabilities, which includes strand-ambiguous SNPs, is the most informative predictor of symptoms of depression. Our findings also highlight the need for a more inclusive approach when generating polygenic risk predictors. This inclusive approach requires allele frequency information from the discovery GWAS. Allele frequency can vary across cohorts with different population structure, thus we recommend that this feature is used across datasets with comparable population structure [13]. We also encourage colleagues to provide allele frequency information in GWAS summary statistics rather than allele frequencies from reference datasets e.g., 1000 genomes project, which will facilitate the more accurate identification of effect alleles across datasets [23, 24].

While PRSoS outperformed PRSice v1.25 for a number of comparisons we do note that PRSice v1.25 calculated PRS more quickly when using the smallest dataset of observed genotypes (Array Data). The difference in performance between PRSice v1.25 and PRSoS when using the array data may reflect the "overhead" i.e., the time taken to parallelize the analysis of PRS when using PRSoS. Likewise, the difference in input file sizes between PRSice v1.25 (.bed file size $=0.03 \mathrm{~GB}$ ) and PRSoS (.gen file size $=0.51 \mathrm{~GB}$ ) may also contribute to the differences in performance using the Array Data. We note that PRSice v1.25 shows consistent performance across all numbers of $p$-value thresholds and is likely to outperform PRSoS when generating PRS at higher resolution (e.g., > 200 p-value thresholds). This crossing point likely varies depending on the availability of computational resources (e.g., number of cores and nodes, available memory) and the input data (e.g., file type, number of samples and SNPs). The recent beta release of PRSice-2 written in $\mathrm{C}++$ improves the performance of PRSice [10] to a level somewhat comparable to PRSoS (see

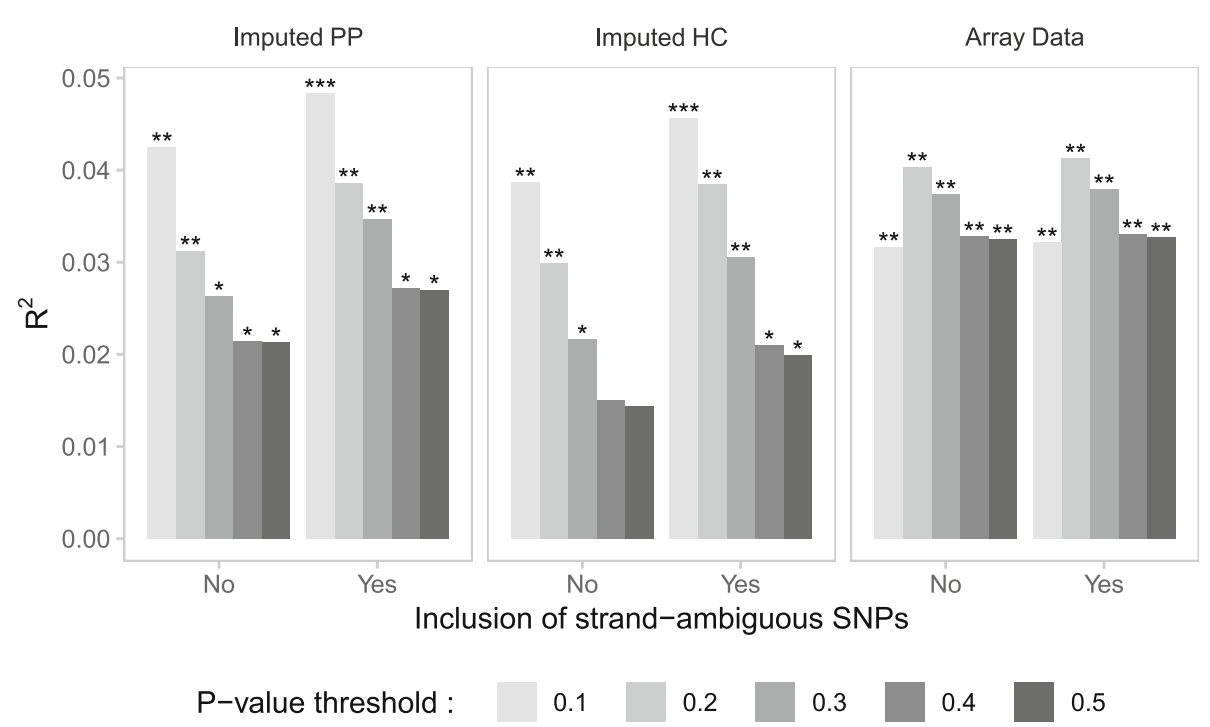

Fig. 5 A PRS for major depressive disorder (MDD) predicts symptoms of depression. Bar plots show the proportion of variance explained by PRS for MDD in the prediction of symptoms of depression. PRS were calculated across three datasets including or excluding strand-ambiguous SNPs at a range of $p$-value thresholds $\left(P_{T}=0.1,0.2,0.3,0.4\right.$, and 0.5$)$. ${ }^{*} p<0.05$, ${ }^{* *} p<0.01,{ }^{* *} p<0.001$. Imputed $\mathrm{PP}=$ imputed posterior probabilities, Imputed $\mathrm{HC}=$ imputed posterior probabilities converted to "hard calls", Array Data = observed genotypes 


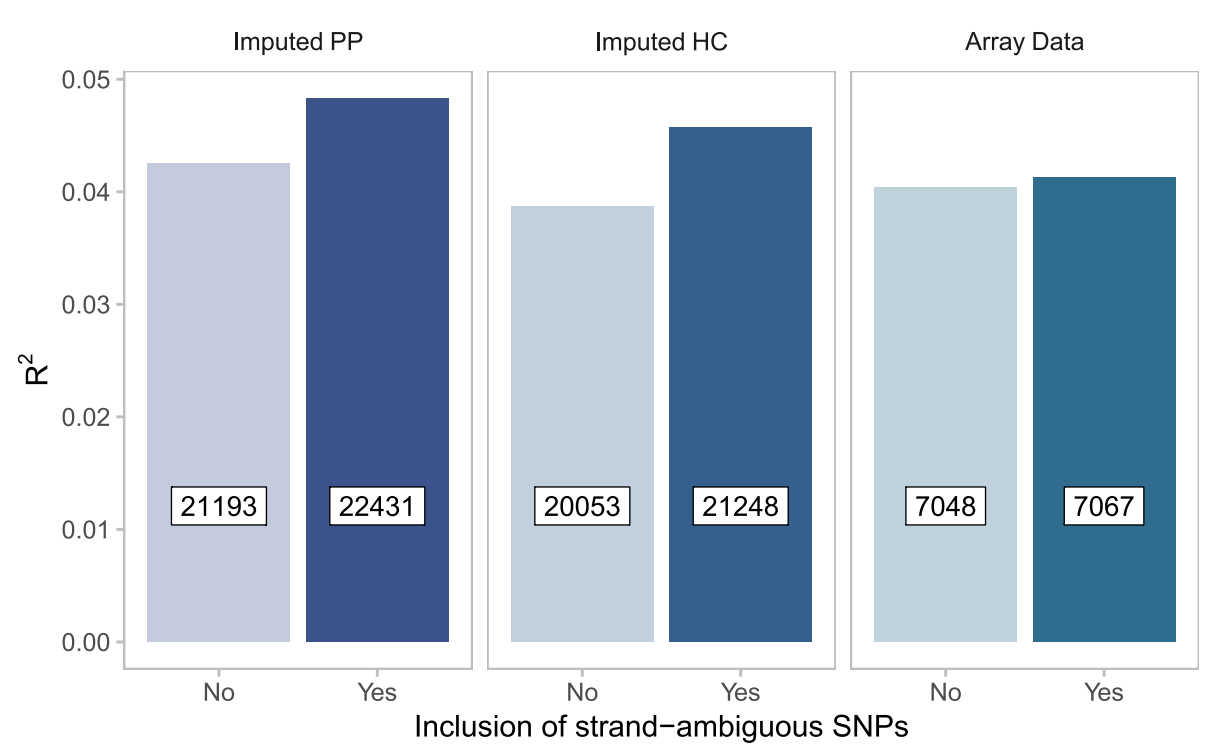

Fig. 6 Best-fit PRS model selection. Bar plots show the proportion of variance in depressive symptoms explained by PRS for major depressive disorder (MDD) as a function of dataset with and without strand-ambiguous SNPs. Only the best-fit models are shown $\left(P_{\mathrm{T}}: \operatorname{Imputed} P P=0.1\right.$, Imputed $\mathrm{HC}=0.1$, Array Data $=0.2$ ). Numbers in boxed inserts refer to the number of SNPs included in each PRS. Imputed PP $=$ imputed posterior probabilities, Imputed HC = imputed posterior probabilities converted to "hard calls", Array Data = observed genotypes

Additional file 3: Figure S2). Despite the enhanced performance of PRSice-2, this new software does not provide the allele frequency function available within PRSoS, which helps to identify effect alleles from stand-ambiguous SNPs across discovery and target datasets.

\section{Conclusions}

We have developed a new software that makes use of parallel computing to accelerate PRS calculation. The increased efficiency of PRSoS and its inclusive approach to strand-ambiguous SNP together with its SNP data output will facilitate the application of PRS to better understand the polygenic basis of complex traits.

\section{Availability and requirements}

Project name: PRS-on-Spark

Project home page: https://github.com/MeaneyLab/ PRSoS

Operating systems: platform independent (tested on Linux CentOS 7 server and Ubuntu 16, MacOS Sierra, and Microsoft Windows 10 standalone computers)

Programming language: Python

Other requirements: Python 2.7, Spark 2.0.0 or higher, Scala 2 or higher, Java 7 or higher, Hadoop 2.6 or higher, Python modules (matplotlib, statsmodels, pandas, numpy)

License: GNU GPL v3, Apache License 2.0

Any restrictions to use by non-academics: None

\section{Additional files}

Additional file 1: Supplementary Methods and Data Analysis. Additional information on the MAVAN cohort. Genetic data quality control and supplementary data analyses are provided. (Additional file 4: Figure S3, Additional file 5: Figure S4). (PDF $142 \mathrm{~kb}$ )

Additional file 2: Figure S1. PRSice v1.25 and PRSoS performance across the number of cores used to generate PRS and five thresholds using the Imputed Hard Call dataset. PRSice v1.25 could only run on 1 core. PRSoS performance was tested with 1, 4, 12, 20, and 24 cores on a Linux CentOS 7, 24-core Intel Xeon server. Error bars indicate standard deviations. (PDF $4 \mathrm{~kb}$ )

Additional file 3: Figure S2. PRSice v1.25, PRSice-2, and PRSOS performance across datasets. Bar plot shows the results of the performance test comparing running PRSice v1.25, PRSice-2, and PRSoS across the datasets. Processing time (y-axis) uses a log base 10 scale. Error bars indicate standard deviations. Numbers in boxed inserts indicate the size of the genotype data input. ${ }^{\dagger}$ Note that the file sizes used for the Imputed PP are same for PRSice v1.25 and PRSoS, thus illustrating the processing speed difference with same file size input. Genotype input formats are different across all three software for the other performance tests. Imputed PP = imputed posterior probabilities, Imputed HC = imputed posterior probabilities converted to "hard calls", Array Data $=$ observed genotypes. (PDF $34 \mathrm{~kb}$ )

Additional file 4: Figure S3. Software performance of generating PRS at five $p$-value thresholds in a single run with different sample sizes. The left panel shows the results using the Imputed Hard Call dataset $(N=264)$. The right panel shows the results using simulated data based on the Imputed Hard Call dataset with five times the sample size $(N=1320)$. Error bars indicate standard deviations. (PDF $4 \mathrm{~kb}$ )

Additional file 5: Figure S4. Software performance between datasets across number of PRS $p$-value thresholds to generate in a single run. Imputed HC = imputed posterior probabilities converted to "hard calls", Array Data $=$ observed genotypes. (PDF $5 \mathrm{~kb}$ )

\section{Abbreviations}

Array Data: Observed genotype dataset; GWAS: Genome-wide association study; Imputed HC: Imputed hard call dataset; Imputed PP: Imputed 
posterior probability dataset; MAVAN: Maternal Adversity, Vulnerability and Neurodevelopment; MDD: Major depressive disorder; PGC: Psychiatric Genomics Consortium; PRS: Polygenic risk scores; PRSoS: PRS-on-Spark; $P_{\text {T: }}$ P-value threshold; SD: Standard deviation; SNP: Single nucleotide polymorphism; Spark: Apache Spark

\section{Acknowledgements}

We thank Jesse Stacey for providing technical support.

\section{Availability of data and materia}

The data that support the findings of this study are available from MAVAN, but restrictions apply to the availability of these data, which were used under license for the current study, and so are not publicly available. Data are, however, available from the authors upon reasonable request and with permission of MAVAN.

Sample test data is provided at: https://github.com/MeaneyLab/PRSoS

\section{Funding}

This work was supported by the Brain Canada Foundation through the Canada Brain Research Fund, Health Canada and Kids Brain Health, the CIFAR Child and Brain Development Program, the Ludmer Centre for Neuroinformatics and Mental Health, and the Sackler Program for Epigenetics \& Psychobiology at McGill University. The funders played no role in the study design, data collection, data analysis or the reporting of these findings.

\section{Authors' contributions}

LMC, NY, EG, TTTN, SAHD, EU, HG, MF, LLM, JLM, MSK, CMTG, PPS, MJM, and KOD contributed to the design of this study. LMC, NY, EG, YZ, TTTN, IP, MF, CMTG, and KOD contributed to the design, development, and testing of the software. LMC and EG performed the statistical analyses. LMC, NY, and YZ tested software's compatibility on other operating systems. LMC and KOD wrote the first draft of the manuscript. All authors read and approved the final manuscript.

\section{Ethics approval and consent to participate}

Fully informed written consent was obtained from participants and ethical approval for this study obtained from the Comité d'éthique de la recherche at the Douglas Hospital Research Centre (Montreal, Canada).

\section{Consent for publication}

Not applicable.

\section{Competing interests}

The authors declare that they have no competing interests.

\section{Publisher's Note}

Springer Nature remains neutral with regard to jurisdictional claims in published maps and institutional affiliations

\section{Author details}

'Douglas Hospital Research Centre, McGill University, H4H1R3, Montreal, Quebec, Canada. ${ }^{2}$ Ludmer Centre for Neuroinformatics and Mental Health, McGill University, Montreal, QC, Canada. ${ }^{3}$ Lady Davis Institute for Medical Research, Jewish General Hospital, Montreal, Quebec, Canada. ${ }^{4}$ Centre for Molecular Medicine and Therapeutics, University of British Columbia, Vancouver, BC, Canada. ${ }^{5}$ Department of Epidemiology, Biostatistics and Occupational Health, McGill University, Montreal, Quebec, Canada. 'Department of Human Genetics, McGill University, Montreal, Quebec, Canada. ${ }^{7}$ Department of Oncology, McGill University, Montreal, Quebec, Canada. ${ }^{8}$ Department of Psychiatry, McGill University, Montreal, Quebec, Canada. ${ }^{9}$ Sackler Program for Epigenetics \& Psychobiology, McGill University, Montreal, Quebec, Canada. ${ }^{10}$ Child and Brain Development Program, Canadian Institute for Advanced Research (CIFAR), Toronto, ON, Canada.

${ }^{11}$ Singapore Institute for Clinical Sciences, Agency for Science, Technology and Research (A*STAR), Singapore, Singapore.
Received: 15 February 2018 Accepted: 18 July 2018

Published online: 08 August 2018

\section{References}

1. Wray NR, Goddard ME, Visscher PM. Prediction of individual genetic risk to disease from genome-wide association studies. Genome Res. 2007;17:1520-8.

2. Krapohl E, Euesden J, Zabaneh D, Pingault J-B, Rimfeld K, von Stumm S, et al. Phenome-wide analysis of genome-wide polygenic scores. Mol Psychiatry. 2016;21:1188-93.

3. Belsky DW, Moffitt TE, Houts R, Bennett GG, Biddle AK, Blumenthal JA, et al. Polygenic risk, rapid childhood growth, and the development of obesity. Arch Pediatr Adolesc Med. 2012;166:515-21.

4. Cross-Disorder Group of the Psychiatric Genomics Consortium. Identification of risk loci with shared effects on five major psychiatric disorders: a genome-wide analysis. Lancet. 2013;381:1371-9.

5. The International Schizophrenia Consortium. Common polygenic variation contributes to risk of schizophrenia and bipolar disorder. Nature. 2009:10:8192.

6. Milaneschi Y, Lamers F, Peyrot WJ, Abdellaoui A, Willemsen G, Hottenga JJ, et al. Polygenic dissection of major depression clinical heterogeneity. Mol Psychiatry. 2016;21:516-22.

7. Sullivan PF, Agrawal A, Bulik CM, Andreassen OA, Børglum AD, Breen G, et al. Psychiatric genomics: an update and an agenda. Am J Psychiatry. 2017:175:15-27.

8. Chang CC, Chow CC, Tellier LCAM, Vattikuti S, Purcell SM, Lee JJ. Secondgeneration PLINK: rising to the challenge of larger and richer datasets. GigaScience. 2015;4:1-16

9. Purcell S, Neale B, Todd-Brown K, Thomas L, Ferreira MAR, Bender D, et al. PLINK: a tool set for whole-genome association and population-based linkage analyses. Am J Hum Genet. 2007:81:559-75.

10. Euesden J, Lewis CM, O'Reilly PF. PRSice: polygenic risk score software. Bioinformatics. 2014;31:1466-8.

11. Conley D, Laidley TM, Boardman JD, Domingue BW. Changing polygenic penetrance on phenotypes in the 20th century among adults in the US population. Sci Rep. 2016:6:30348.

12. Holmes AJ, Lee PH, Hollinshead MO, Bakst L, Roffman JL, Smoller JW, Buckner RL. Individual differences in amygdala-medial prefrontal anatomy link negative affect, impaired social functioning, and polygenic depression risk. J Neurosci. 2012;32:18087-100.

13. Martin AR, Gignoux CR, Walters RK, Wojcik GL, Neale BM, Gravel S, et al. Human demographic history impacts genetic risk prediction across diverse populations. Am J Hum Genet. 2017;100:635-49.

14. Whalley HC, Sprooten E, Hackett S, Hall L, Blackwood DH, Glahn DC, et al. Polygenic risk and white matter integrity in individuals at high risk of mood disorder. Biol Psychiatry. 2013;74:280-6.

15. Vilhjálmsson BJ, Yang J, Finucane HK, Gusev A, Lindström S, Ripke S, et al. Modeling linkage disequilibrium increases accuracy of polygenic risk scores. Am J Hum Genet. 2015:97:576-92.

16. Major Depressive Disorder Working Group of the Psychiatric GWAS Consortium. A mega-analysis of genome-wide association studies for major depressive disorder. Mol Psychiatry. 2013;18:497-511.

17. Psychiatric GWAS Consortium Bipolar Disorder Working Group. Large-scale genome-wide association analysis of bipolar disorder identifies a new susceptibility locus near ODZ4. Nat Genet. 2011;43:977-83.

18. Locke AE, Kahali B, Berndt SI, Justice AE, Pers TH, Day FR, et al. Genetic studies of body mass index yield new insights for obesity biology. Nature. 2015:518:197-206.

19. Stein MB, Chen C-Y, Ursano RJ, Cai T, Gelernter J, Heeringa SG, et al. Genome-wide association studies of posttraumatic stress disorder in 2 cohorts of US army soldiers. JAMA Psychiatry. 2016;73:695-704.

20. O'Donnell KA, Gaudreau H, Colalillo S, Steiner M, Atkinson L, Moss E, et al. The maternal adversity, vulnerability and neurodevelopment project: theory and methodology. Can J Psychiatr. 2014:59:497-508.

21. Radloff LS. The CES-D scale: a self-reported depression scale for research in the general population. Appl Psychol Meas. 1977;1:385-401.

22. Wray NR, Lee SH, Mehta D, Vinkhuyzen AAE, Dudbridge F, Middeldorp CM. Research review: polygenic methods and their application to psychiatric traits. J Child Psychol Psychiatry Allied Disciplines. 2014;55:1068-87.

23. Sand PG. A lesson not learned: allele misassignment. Behav Brain Funct. 2007;3:65

24. The 1000 Genomes Project Consortium. A global reference for human genetic variation. Nature. 2015:526:68 